\title{
Funciones compatibles de ahusamiento-volumen para tres especies de Pinus en la Unidad de Manejo Forestal 0808 del estado de Chihuahua
}

\author{
Taper-volume compatible functions for three Pinus species at \\ Management Forest Unit 0808 from Chihuahua state
}

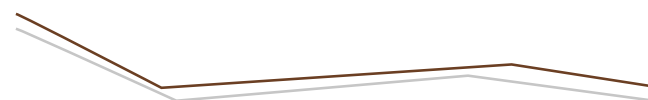

Edgar Silva-González*, Marco Antonio Nava-Moreno*, Francisco Javier Hernández**凶, José Guadalupe Colín**

Silva-González, E., Nava-Moreno, M. A., Hernández, F. J., \& Colín, J. G. (2018). Funciones compatibles de ahusamiento-volumen para tres especies de Pinus en la Unidad de Manejo Forestal 0808 del estado de Chihuahua. Investigación y Ciencia de la Universidad Autónoma de Aguascalientes, 26(73), 58-67.
\end{abstract}

RESUMEN

El propósito del presente estudio fue ajustar nueve modelos compatibles de ahusamiento-volumen para las especie de Pinus arizonica, $P$. durangensis y $P$. engelmannii del estado de Chihuahua. Para realizar este estudio se seleccionaron 111 árboles para la primera especie, 108 para la segunda y 91 para la tercera. El análisis implicó la incoporación del modelo de volumen de Schumacher \& Hall a cada uno de los nueve modelos de ahusamiento para ajustarlos de manera simultánea y seleccionar la mejor función compatible de ahusamientovolumen. Los resultados indicaron que los modelos de ahusamiento-volumen con mejor ajuste fueron el de Fang y el de Cruz-Cobos para las tres especies.

\section{ABSTRACT}

The purpose of the present study was to fit nine taper-volume compatible models to Pinus arizonica, $P$. durangensis and $P$. engelmannii species from Chihuahua State, Mexico. To realize this study, 111 ,

Palabras clave: ahusamiento-volumen; Schumacher; Pinus arizonica; Pinus durangensis; Pinus engelmannii. Keywords: taper-volumen; Schumacher; Pinus arizonica; Pinus durangensis; Pinus engelmannii.

\section{Recibido: 4 de abril de 2017, aceptado: 31 de octubre de 2017}

* Proceso Tesis Instituto Tecnológico de El Salto. Mesa del Tecnológico s/n, C. P. 34942, El Salto, Durango, México. Correo electrónico: edgar silva7@hotmail.com; navamm_91@hotmail.com

** División de Estudios de Posgrado, Instituto Tecnológico de El Salto. Mesa del Tecnológico s/n, C. P. 34942, El Salto, Durango, México. Correo electrónico: fcojhernan@yahoo.com.mx; jose colin8@hotmail.com

$凶$

Autor para correspondencia
108 and 91 trees of each species were selected, respectively. The analysis required linking the Schmacher \& Hall volume model to each one of nine taper models to fit them simultaneously and select the most compatible taper-volume model. The best results for the three pine species were obtained with the compatible taper-volume models proposed by Fang and Cruz-Cobos.

INTRODUCCIÓN

Los esfuerzos realizados para lograr una producción constante y balanceada de los recursos forestales maderables inducen a que el administrador forestal cuente con herramientas matemáticas que estimen a un buen nivel de precisión tanto el volumen total a extraer como los volúmenes destinados a la elaboración de productos primarios, secundarios y leñas (Valdez-Lazalde \& Lynch, 2000). La cuantificación precisa de las existencias reales volumétricas totales y del volumen comercial es un respaldo importante en la toma de decisiones sobre las prescripciones silvícolas. El volumen total y parcial del fuste se puede estimar directamente a través de ecuaciones independientes de volumen total y comercial (Clutter, 1980; Demaerschalk, 1972; Lynch, Chang, \& Chandler, 1992), ecuaciones de razon (Teshome, 2005), mediante la integración matemática del volumen acumulado a las diversas dimensiones comerciales del fuste aplicando las ecuaciones de ahusamiento previamente ajustadas o a través de la inclusión de ecuaciones de volumen a las de ahusamiento para ajustarlas de manera simultánea y generar funciones compatibles de ahusamiento-volumen (Fang, Borders, \& Bailey, 2000). 


\section{IIVESTIGAGIÓn Y CUERCIA DE LA UกIVERSIDAD AUTÓnOMA DE RGUASCALIERTES}

Aunque a través del tiempo se han desarrollado una serie de ecuaciones matemáticas y de regresión para estimar el volumen total y comercial del bosque aplicadas a las especies de la región forestal donde se desarrolló este estudio (Pompa-García, CorralRivas, Díaz-Vázquez, \& Martínez-Salvador, 2009), se especula que muchas de ellas no se han aplicado de manera compatible y otras han perdido precisión debido a los cambios estructurales de la vegetación arbórea. Esas ecuaciones fueron diseñadas para aplicarse a bosques naturales originales con características dimensionales muy superiores a las que actualmente tienen los bosques de segundo y tercer crecimiento. Al considerar lo anterior y con el propósito de actualizar y contribuir a la estimación precisa de las existencias totales y comerciales del volumen total y comercial de las especies forestales que se desarrollan en el estado de Chihuahua y así garantizar una producción sostenible, el presente trabajo se dirigió a ajustar diversos modelos de volumen y ahusamiento y definir el mejor para determinar, a partir de ecuaciones simultáneas, una ecuación compatible de ahusamiento-volumen que permita estimar el volumen total y comercial de Pinus arizonica, $P$. durangensis y $P$. engelmannii de la Unidad de Manejo Forestal 0808 (UMAFOR 0808) del estado de Chihuahua.

MATERIALES Y MÉTODOS

\section{Descripción del área de estudio}

El presente estudio se llevó a cabo dentro del municipio de Guadalupe y Calvo, el cual se encuentra ubicado en la parte sur del estado de Chihuahua dentro de la Sierra Madre Occidental, específicamente en la zona denominada Sierra Tarahumara. Este municipio limita al noroeste con los municipios de Morelos y Balleza, al norte con el municipio de Guachochi. Al sur y al sureste colinda con el estado de Durango, en particular con los municipios de Guanacevi, Tepehuanes y Tamazula. Al suroeste con el municipio de Badiraguato del estado de Sinaloa (figura 1). El municipio comprende ocho comunidades, 26 ejidos y 126 predios particulares que se encuentran bajo manejo forestal, cubriendo una superficie total de 904,780 ha. Los principales climas que caracterizan a la región pertenecen a los grupos CW, Acw y Bs que corresponden a los templados subhúmedos, semicálidos y semisecos, respectivamente. Las precipitaciones promedio en la región del bosque templado varían de 500 a 1050 $\mathrm{mm}$, mientras que las temperaturas medias van de los 16 a los $20^{\circ} \mathrm{C}$. La mínima y máxima extrema en la región es de -15 y $37^{\circ} \mathrm{C}$.

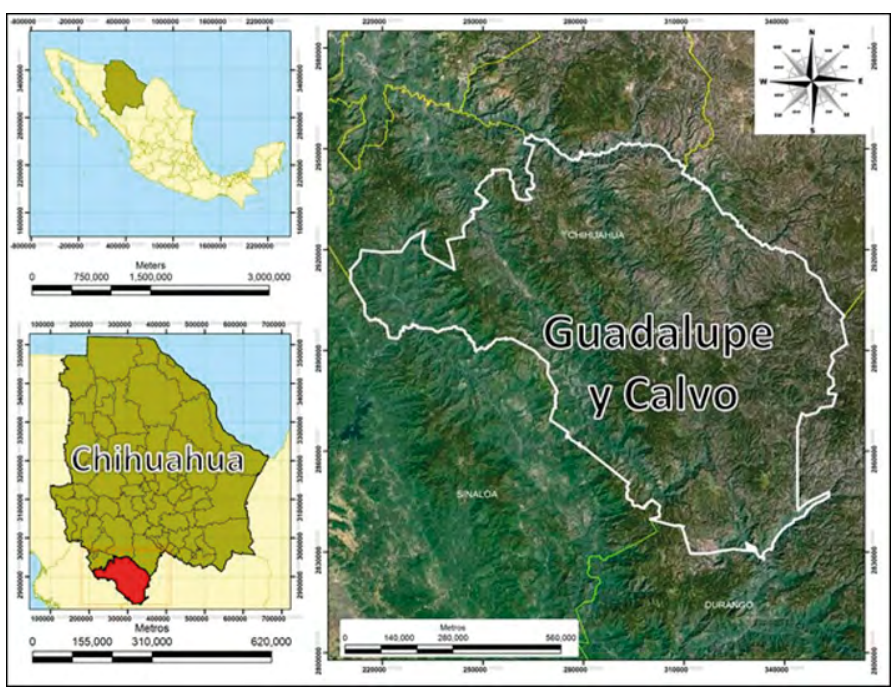

Figura 1. Ubicación del área de estudio.

Elaboración propia.

\section{Muestreo}

Para el presente estudio se seleccionaron 111 árboles de Pinus arizonica, 108 de $P$. durangensis y 91 de $P$. engelmannii considerando su conformación morfológica y estado de salud. La muestra representó a todas las categorías diamétricas presentes en el área de estudio. Los árboles seleccionados fueron identificados por especie para ser derribados, desramados y seccionados en trozas de diferentes dimensiones (figuras 2 y 3). De cada árbol se midió y registro la siguiente información: diámetro y altura del tocón, diámetro a 0.30 m y 0.60 m después de la altura del tocón, así como diámetro a una altura de $1.30 \mathrm{~m}$ a partir del suelo. Posteriormente se registraron los diámetros a cada $2.60 \mathrm{~m}$ hasta llegar a la parte de la punta del árbol.

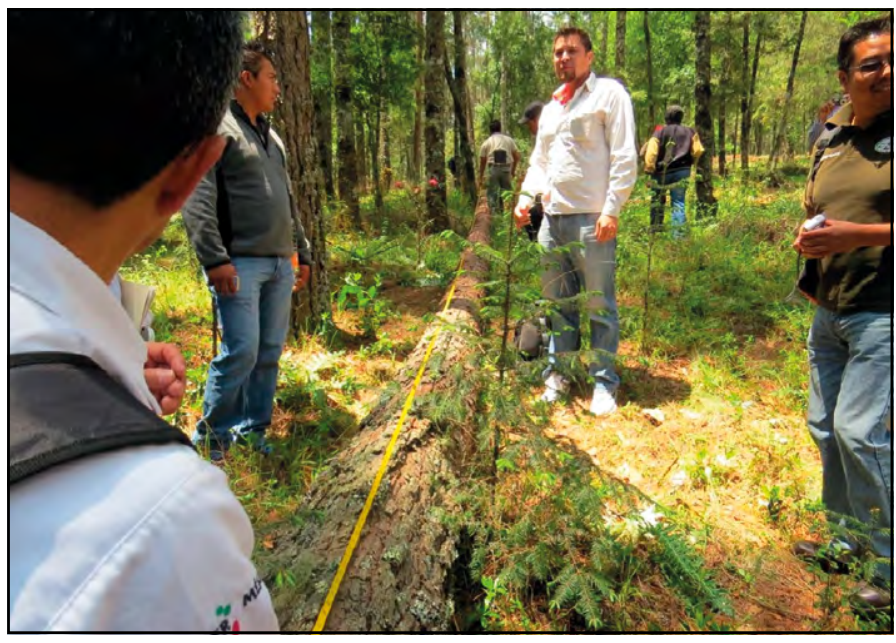

Figura 2. Medición de los árboles derribados.

Fotografía proporcionada por Francisco Javier Hernández. 


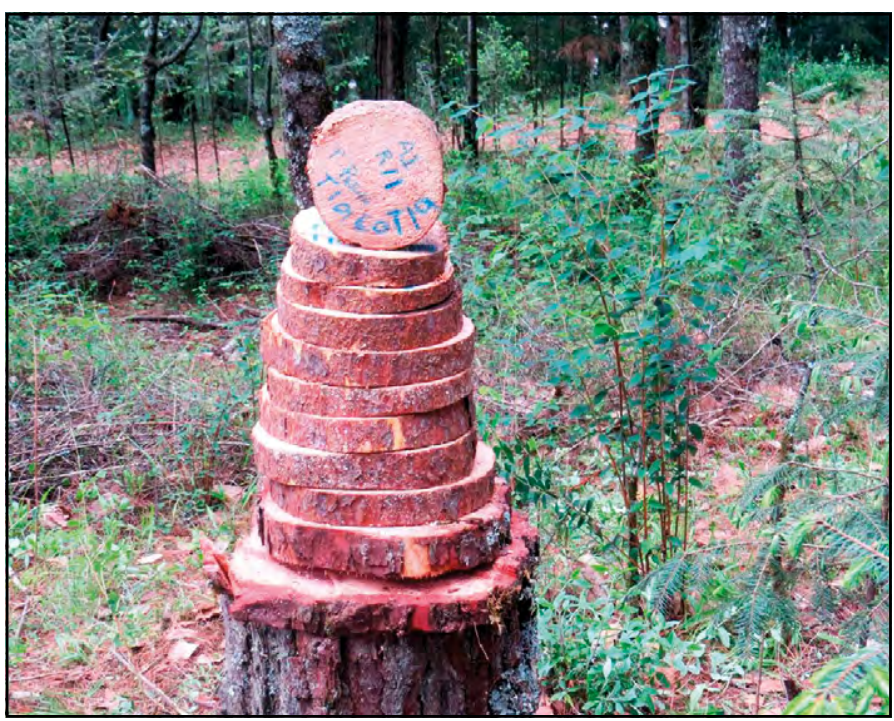

Figura 3. Rodajas que representan el perfil del fuste del arbolado. Fotografía proporcionada por Francisco Javier Hernández.

\section{Cubicación del arbolado}

Con la información de campo, se estimó en cada árbol el volumen del tocón, trozas del fuste y troza de la punta utilizando las fórmulas de cilindro, Smalian y del cono, respectivamente (tabla 1).

Tabla 1

Fórmulas para cubicación de trozas

\begin{tabular}{cc}
\hline Nombre & Ecuación \\
\hline Cilindro & $V=\frac{\pi}{4}\left(D t^{2} \mathrm{H}\right)$ \\
\hline Smalian & $V=\frac{\left(S_{1}+S_{2}\right)}{2} \mathrm{H}$ \\
\hline Cono & $V=\frac{\pi D b^{2}}{12} H$ \\
\hline
\end{tabular}

Nota: Elaboración propia.

donde:

$V=$ volumen de la troza $\left(\mathrm{m}^{3}\right)$

$\mathrm{H}=$ longitud de la troza $(\mathrm{m})$

$\mathrm{S}_{1}=$ área basal mayor de cada troza $\left(\mathrm{m}^{2}\right)$

$\mathrm{S}_{2}=$ área basal menor de cada troza $\left(\mathrm{m}^{2}\right)$

Dł= diámetro del tocón $(\mathrm{m})$

$\mathrm{Db}=$ diámetro de la base de la punta (m)

El volumen total del árbol se definió como la suma de los volúmenes de cada una de las secciones del árbol.

$$
v_{t}=\sum_{i=I}^{k}\left(V_{1}+V_{2}+V_{3}+\ldots .+V_{n}\right)
$$

donde:

$\mathrm{V} t=$ volumen total del fuste limpio

$\mathrm{V}_{\mathrm{i}}=$ volumen de cada troza

\section{Modelos Ajustados}

Primero se ajustaron de manera independiente, el modelo de volumen total de Schumacher \& Hall, cuya expresión exponencial es:

$$
V=\alpha_{0} D_{n}^{\alpha_{1}} H^{\alpha_{2}}
$$

donde:

$$
\begin{aligned}
& V=\text { volumen total } \\
& D_{n}=\text { diámetro normal } \\
& H=\text { altura total } \\
& \alpha_{i}=\text { parámetros }
\end{aligned}
$$

y los de ahusamiento a cada una de las tres especies evaluadas para determinar y justificar la bondad de ajuste de sus parámetros y su aplicabilidad a las especies estudiadas. Posteriormente, se ajustaron de manera simultánea cada uno de los modelos de ahusamiento con el de volumen para generar las funciones compatibles ahusamiento-volumen (tabla 2) y así poder determinar el volumen total o comercial a cualquier altura o diámetro fijado.

El ajuste de los modelos se realizó utilizando el software SAS Institute 9.0 mediante una estimación simultánea a través de máxima verosimilitud con información completa (FIML). Borders (1989) y Fang et al. (2000), señalan que el ajuste con FIML homogeniza y minimiza el error estándar de los parámetros en el sistema y además permite la compatibilidad completa del sistema de ahusamiento y volumen. Durante el ajuste de los modelos compatibles ahusamiento-volumen, se realizó la prueba de autocorrelación de Dublin Watson y la corrección del efecto a través del modelo autorregresivo de segundo orden (CAR2). Para evitar problemas en la estimación de los parámetros debido a que el diámetro de la punta del fuste es igual a cero, se aplicó un valor pequeño en este punto. 


\section{IIVESTIGAGIÓn Y CHERGIA DE LA UחIVERSIDAD AUTÓNOMA DE AGUASCALIERTES}

Tabla 2

Modelos compatibles de ahusamiento volumen aplicados

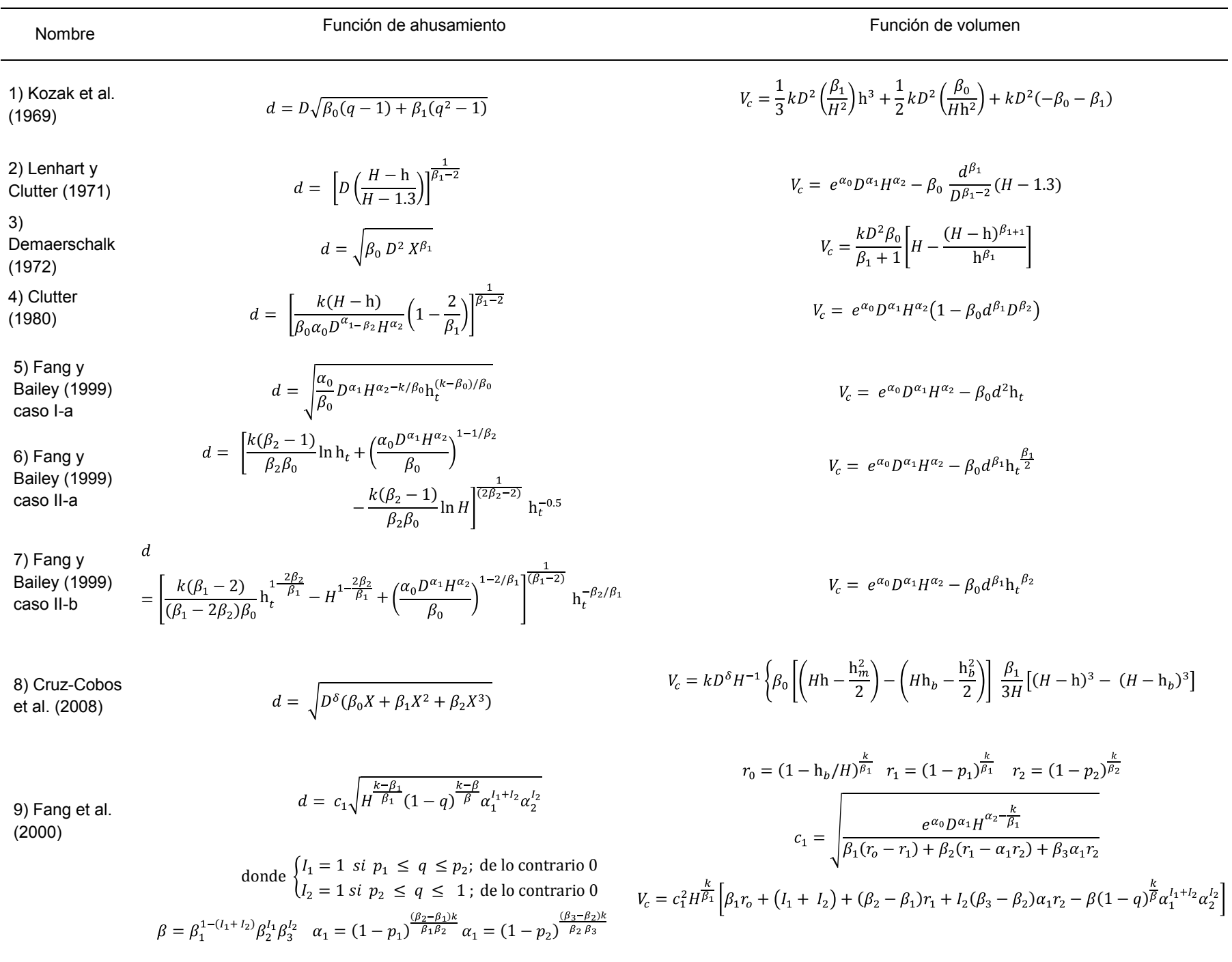

Nota: Donde $D=$ diámetro normal $(\mathrm{m}) ; d=$ diámetro $(\mathrm{cm})$ a la altura $h(\mathrm{~m})$ desde el nivel del tocón; $H=$ altura total de los arboles $(\mathrm{m}) ; h_{b}=$ altura del tocón $(\mathrm{m}) ; V c=$ volumen comercial variable en $\left(\mathrm{m}^{3}\right) ; k=\pi / 40000 ; \alpha_{i}, \beta_{i}, \delta, \mathrm{p}_{1}, \mathrm{p}_{2}=$ coeficientes a determinar mediante el ajuste; $X=(H-h) / H ; z_{1}=h_{b} / H ; z_{b}=\left(H-h_{b}\right) / H$. Elaboración propia.

La calificación de la bondad de ajuste de los modelos se realizó utilizando los estadísticos coeficiente de determinación ajustado $\left(R^{2} a d j\right)$ y raíz del cuadrado medio del error (REMC):

$R^{2} a d j=1-\left[\frac{\sum_{n=1}^{n}\left(Y_{i}-\widehat{Y}_{l}\right)^{2}}{\sum_{i=1}^{n}\left(Y_{i}-\bar{Y}_{l}\right)^{2}}\right]\left(\frac{n-1}{n-p}\right) \quad R E M C=\sqrt{\frac{\left[\sum_{i=1}^{n}\left(Y_{i}-\widehat{Y}_{l}\right)^{2}\right]}{n-p}}$

donde:

$\widehat{Y}_{l}, \bar{Y}_{l} y Y_{i}=$ valor predicho, media y valor observado de la variable dependiente

$n=$ número de observaciones

$p=$ número de parámetros del modelo.
RESULTADOS

Para corroborar que la tendencia de los datos sea la que presenta el ahusamiento del fuste de los árboles y detectar valores atípicos en la base de datos que se utilizó, se graficaron los diámetros y los volúmenes acumulados contra las alturas del fuste. En las figuras 4, 5, y 6 se observa la inexistencia de datos atípicos y la tendencia del perfil del fuste y el volumen de los árboles en relación con la altura de cada una de las especies estudiadas.

En la tabla 3 se presentan los estimadores descriptivos número de árboles, valor promedio y 
IIVESTIGACIÓn Y CIERCIA DE LA UחIVERSIDAD AUTÓกOMA DE RGUASCALIERTES valores mínimo y máximo de las variables diámetro normal, altura total y volumen fustal de Pinus arizonica, $P$. durangensis, y $P$. engelmannii.

Aunque todos los modelos compatibles ahusamiento-volumen para estimar volumen comercial fueron significativos, los que mejor se ajustaron a las tres especies fueron los de Fang et al. (2000) y los de Cruz-Cobos, De los Santos-Posadas y Valdez-Lazalde (2008). Los coeficientes de determinación estimados para $P$. arizónica $\left(R^{2}=0.97\right)$ y $P$. durangensis $\left(R^{2}=0.99\right)$ fueron similares en ambos modelos. En relación con $P$. engelmanii, el modelo de Fang et al. (2000) estima un $R^{2}$ igual a 0.98 y el de Cruz-Cobos et al. (2008) un valor de 0.97. La evaluación de la REMC para estos mismos modelos señala que en $P$. durangensis y $P$. engelmanii la función de volumen comercial de Fang et al. (2000) fue ligeramente mejor a la de Cruz-Cobos et al. (2008) (tabla 4).

Al igual que con los modelos de ahusamientovolumen para estimar volumen comercial, todos los modelos para estimar el ahusamiento presentaron buen ajuste, enfatizando que, nuevamente, los mejores modelos de ahusamiento fueron los de Fang et al. (2000) y Cruz-Cobos et al. (2008) (tabla 4). Estos modelos se aplican a la estimación del diámetro a cualquier altura o la altura a cualquier diámetro del arbolado en las tres especies.
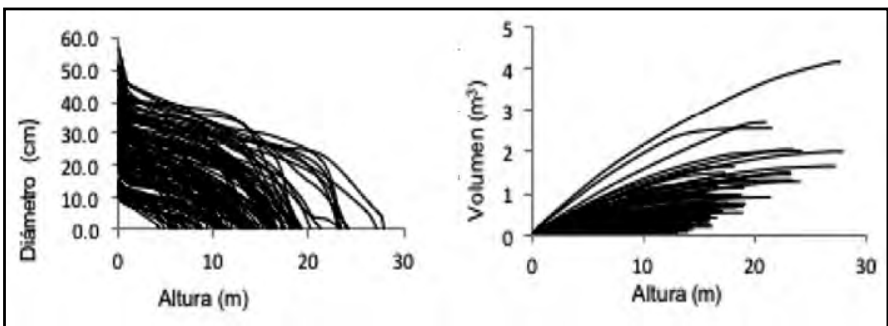

Figura 4. Diámetros y volúmenes acumulados en función de la altura para Pinus arizonica.

Elaboración propia.
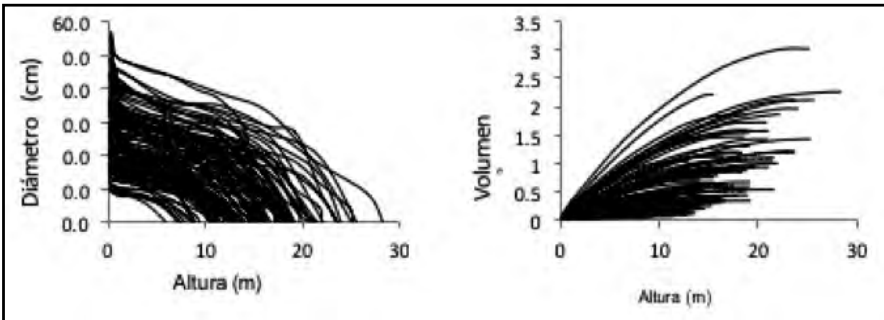

Figura 5. Diámetros y volúmenes acumulados en función de la altura para Pinus durangensis.

Elaboración propia.
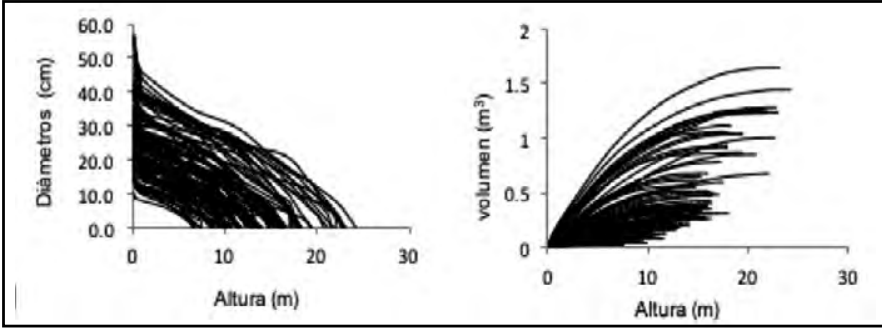

Figura 6. Diámetros y volúmenes acumulados en función de la altura para Pinus engelmanii.

Elaboración propia.
El análisis de significancia de los estimadores de los parámetros relacionados con el ahusamiento indica que en el modelo compatible de Fang et al. (2000), ajustado a P. arizonica, B1 careció de significancia (tabla 5). De manera similar, en el modelo caso II-b de Fang y Bailey (1999), Bo y $\mathrm{B} 1$ no fueron significativos en Pinus durangensis y $P$. engelmannii, respectivamente. En el modelo de Cruz-Cobos et al. (2008), B1 no fue significativo para $P$. engelmannii (tablas 6 y 7 ).
Tabla 3

Valores estadísticos descriptivos de los datos utilizados

\begin{tabular}{llcccc}
\hline Variable & \multicolumn{1}{c}{ Especie } & $\mathrm{N}$ & Media & Mínimo & Máximo \\
\hline \multirow{2}{*}{ Dn $(\mathrm{cm})$} & P. arizonica & 111 & 25.87 & 7.7 & 59 \\
& P. durangensis & 108 & 28.2 & 9.5 & 55 \\
& P. engelmannii & 91 & 23.94 & 8.1 & 48 \\
& P. arizonica & 111 & 14.43 & 4.36 & 28 \\
$\mathrm{H}(\mathrm{m})$ & P. durangensis & 108 & 15.7 & 6.05 & 25.9 \\
& P. engelmannii & 91 & 14.11 & 6.62 & 24.27 \\
$\mathrm{~V}\left(\mathrm{~m}^{3}\right)$ & P. arizonica & 111 & 0.6022 & 0.0048 & 4.1848 \\
& P. durangensis & 108 & 0.6707 & 0.0305 & 3.0248 \\
& P. engelmannii & 91 & 0.4102 & 0.0198 & 1.635 \\
\hline
\end{tabular}

Nota: Donde $D n$ es el diámetro normal; $H$ es la altura total; $V$ es el volumen total; $N$ es el número de árboles utilizados.

Elaboración propia. 
Tabla 4

Estadísticos de ajuste de los sistemas probados ahusamiento-volumen para Pinus arizonica, P. durangensis y P. engelmannii

\begin{tabular}{|c|c|c|c|c|c|}
\hline \multirow{2}{*}{ Sistema de volumen compatible } & \multirow{2}{*}{ Especie } & \multicolumn{2}{|c|}{$\begin{array}{c}\text { Ecuación de volumen } \\
\text { comercial }\end{array}$} & \multicolumn{2}{|c|}{ Ahusamiento } \\
\hline & & REMC & $\mathrm{R}^{2} \mathrm{adj}$ & REMC & $R^{2} a d j$ \\
\hline & P. arizonica & 0.1135 & 0.95 & 3.1368 & 0.95 \\
\hline \multirow[t]{3}{*}{ Kozak et al. (1969) } & P. durangensis & 0.0710 & 0.98 & 2.8084 & 0.96 \\
\hline & P. engelmannii & 0.0815 & 0.96 & 4.1239 & 0.89 \\
\hline & P. arizonica & 0.2624 & 0.74 & 3.4674 & 0.94 \\
\hline \multirow[t]{3}{*}{ Lenhart y Clutter (1971) } & P. durangensis & 0.1895 & 0.84 & 3.0097 & 0.95 \\
\hline & P. engelmannii & 0.2048 & 0.72 & 4.9495 & 0.85 \\
\hline & P. arizonica & 0.0710 & 0.97 & 2.8501 & 0.96 \\
\hline \multirow[t]{3}{*}{ Demaerschalk (1972) } & P. durangensis & 0.1103 & 0.97 & 2.9311 & 0.96 \\
\hline & P. engelmannii & 0.0815 & 0.97 & 4.1592 & 0.89 \\
\hline & P. arizonica & 0.2477 & 0.76 & 3.4499 & 0.94 \\
\hline \multirow[t]{3}{*}{ Clutter (1980) } & P. durangensis & 0.1800 & 0.86 & 3.1998 & 0.95 \\
\hline & P. engelmannii & 0.1877 & 0.77 & 4.3856 & 0.88 \\
\hline & P. arizonica & 0.1761 & 0.88 & 3.1709 & 0.95 \\
\hline \multirow[t]{3}{*}{ Fang y Bailey (1999) caso I-a } & P. durangensis & 0.1278 & 0.93 & 2.8357 & 0.96 \\
\hline & P. engelmannii & 0.1340 & 0.88 & 3.9439 & 0.90 \\
\hline & P. arizonica & 0.1713 & 0.89 & 3.1876 & 0.95 \\
\hline \multirow[t]{3}{*}{ Fang y Bailey (1999) caso II-a } & P. durangensis & 0.1090 & 0.94 & 2.7806 & 0.96 \\
\hline & P. engelmannii & 0.1226 & 0.90 & 3.7477 & 0.91 \\
\hline & P. arizonica & 0.1641 & 0.90 & 3.2480 & 0.94 \\
\hline \multirow[t]{3}{*}{ Fang y Bailey (1999) caso II-b } & P. durangensis & 0.1103 & 0.95 & 2.9311 & 0.96 \\
\hline & P. engelmannii & 0.1123 & 0.92 & 3.8970 & 0.91 \\
\hline & P. arizonica & 0.0841 & 0.97 & 2.7568 & 0.96 \\
\hline \multirow[t]{3}{*}{ Cruz-Cobos et al. (2008) } & P. durangensis & 0.0565 & 0.99 & 2.4080 & 0.97 \\
\hline & P. engelmannii & 0.0460 & 0.97 & 2.3390 & 0.96 \\
\hline & P. arizonica & 0.0880 & 0.97 & 2.4950 & 0.97 \\
\hline \multirow[t]{2}{*}{ Fang et al. (2000) } & P. durangensis & 0.0536 & 0.99 & 1.9540 & 0.98 \\
\hline & P. engelmannii & 0.0520 & 0.98 & 1.8230 & 0.98 \\
\hline
\end{tabular}

Nota: Elaboración propia. 
Tabla 5

Datos descriptivos de los modelos compatibles de volumen comercial-ahusamiento para P. arizonica

\begin{tabular}{|c|c|c|c|c|c|c|c|c|c|c|c|}
\hline $\begin{array}{l}\text { Sistema de } \\
\text { volumen }\end{array}$ & & $\alpha_{0}$ & $\alpha_{1}$ & $\alpha_{2}$ & $\beta_{0}$ & $\beta_{1}$ & $\beta_{2}$ & $\beta_{3}$ & $\rho_{1}$ & $\rho_{2}$ & $\delta$ \\
\hline & $\Psi$ & & & & & -1.41105 & 0.246205 & & & & \\
\hline \multirow[t]{3}{*}{ 1) } & E & & & & & 0.0277 & 0.0215 & & & & \\
\hline & $\infty$ & & & & & $<.0001$ & $<.0001$ & & & & \\
\hline & $\Psi$ & -10.1233 & 1.793806 & 1.211452 & 0.000026 & 3.568662 & & & & & \\
\hline \multirow[t]{3}{*}{ 2) } & E & 0.0973 & 0.0212 & 0.0287 & $2.016 \times 10^{-7}$ & 0.0139 & & & & & \\
\hline & $\infty$ & $<.0001$ & $<.0001$ & $<.0001$ & $<.0001$ & $<.0001$ & & & & & \\
\hline & $\Psi$ & & & & & 1.147186 & 1.111923 & & & & \\
\hline \multirow[t]{3}{*}{ 3) } & E & & & & & 0.00518 & 0.0109 & & & & \\
\hline & $\infty$ & & & & & $<.0001$ & $<.0001$ & & & & \\
\hline & $\Psi$ & -9.34291 & 1.857118 & 0.921897 & 0.331701 & 3.432083 & 3.248107 & & & & \\
\hline \multirow[t]{3}{*}{ 4) } & E & 0.0667 & 0.0178 & 0.0212 & 0.0246 & 0.0113 & 0.0190 & & & & \\
\hline & $\infty$ & $<.0001$ & $<.0001$ & $<.0001$ & $<.0001$ & $<.0001$ & $<.0001$ & & & & \\
\hline & $\Psi$ & -9.75876 & 1.830664 & 1.101103 & 0.000036 & & & & & & \\
\hline \multirow[t]{3}{*}{ 5) } & E & 0.0475 & 0.0129 & 0.0168 & $1.584 \times 10^{-7}$ & & & & & & \\
\hline & $\infty$ & $<.0001$ & $<.0001$ & $<.0001$ & $<.0001$ & & & & & & \\
\hline & $\Psi$ & -9.65723 & 1.806518 & 1.095294 & 0.000043 & 1.961517 & & & & & \\
\hline \multirow[t]{3}{*}{ 6) } & E & 0.0520 & 0.0130 & 0.0163 & $1.075 \times 10^{-6}$ & 0.00463 & & & & & \\
\hline & $\infty$ & $<.0001$ & $<.0001$ & $<.0001$ & $<.0001$ & $<.0001$ & & & & & \\
\hline & $\Psi$ & -9.48577 & 1.693734 & 1.174786 & 0.000059 & 1.766681 & 1.120859 & & & & \\
\hline \multirow[t]{3}{*}{ 7) } & E & 0.0549 & 0.0187 & 0.0189 & $2.833 \times 10^{-6}$ & 0.0257 & 0.0194 & & & & \\
\hline & $\infty$ & $<.0001$ & $<.0001$ & $<.0001$ & $<.0001$ & $<.0001$ & $<.0001$ & & & & \\
\hline & $\psi$ & & & & 1.756762 & -1.33616 & 1.213476 & & & & 1.917957 \\
\hline \multirow[t]{3}{*}{ 8) } & E & & & & 0.788 & 0.1412 & 0.1119 & & & & 0.0111 \\
\hline & $\infty$ & & & & $<.0001$ & $<.0001$ & $<.0001$ & & & & $<.0001$ \\
\hline & $\Psi$ & -9.91486 & 1.978935 & 0.98201 & & $7.882 \times 10^{-6}$ & 0.000039 & 0.133998 & 0.039451 & 0.99625 & \\
\hline \multirow[t]{2}{*}{ 9) } & $E$ & 0.0520 & 0.0146 & 0.0137 & & $7.001 \times 10^{-7}$ & $1.75 \times 10^{-7}$ & 0.0116 & 0.00287 & 0.0 & \\
\hline & $\infty$ & $<.0001$ & $<.0001$ & $<.0001$ & & $<.0001$ & $<.0001$ & 0.1718 & $<.0001$ & $<.0001$ & \\
\hline
\end{tabular}


IIVESTIGACIÓn Y CIEnCIR DE LA UNIVERSIDAD AUTÓnOMF

DE RGUASCALIETTES

Tabla 6

Datos descriptivos de los modelos compatibles de volumen comercial-ahusamiento para P. durangensis

\begin{tabular}{|c|c|c|c|c|c|c|c|c|c|c|c|}
\hline $\begin{array}{l}\text { Sistema de } \\
\text { volumen }\end{array}$ & & $\alpha_{0}$ & $\alpha_{1}$ & $\alpha_{2}$ & $\beta_{0}$ & $\beta_{1}$ & $\beta_{2}$ & $\beta_{3}$ & $\rho_{1}$ & $\rho_{2}$ & $\delta$ \\
\hline & $\psi$ & & & & & -1.49276 & 0.358556 & & & & \\
\hline \multirow[t]{3}{*}{ 1) } & E & & & & & 0.0226 & 0.0173 & & & & \\
\hline & $\infty$ & & & & & $<.0001$ & $<.0001$ & & & & \\
\hline & $\psi$ & -10.2683 & 2.165869 & 0.800167 & 0.000027 & 3.528008 & & & & & \\
\hline \multirow[t]{3}{*}{ 2) } & $E$ & 0.0826 & 0.0212 & 0.0156 & $1.344 \times 10^{-7}$ & 0.0140 & & & & & \\
\hline & $\infty$ & $<.0001$ & $<.0001$ & $<.0001$ & $<.0001$ & $<.0001$ & & & & & \\
\hline & $\psi$ & & & & & 1.111322 & 1.188438 & & & & \\
\hline \multirow[t]{3}{*}{ 3) } & E & & & & & 0.00462 & 0.00995 & & & & \\
\hline & $\infty$ & & & & & $<.0001$ & $<.0001$ & & & & \\
\hline & $\Psi$ & -10.1466 & 2.08043 & 0.893011 & 1.040669 & 3.354161 & 3.467695 & & & & \\
\hline \multirow[t]{3}{*}{ 4) } & E & 0.0747 & 0.0197 & 0.0145 & 0.0863 & 0.0104 & 0.0247 & & & & \\
\hline & $\infty$ & $<.0001$ & $<.0001$ & $<.0001$ & $<.0001$ & $<.0001$ & $<.0001$ & & & & \\
\hline & $\Psi$ & -9.95119 & 2.145739 & 0.758376 & 0.000034 & & & & & & \\
\hline \multirow[t]{3}{*}{ 5) } & E & 0.0505 & 0.0146 & 0.0106 & $1.571 \times 10^{-7}$ & & & & & & \\
\hline & $\infty$ & $<.0001$ & $<.0001$ & $<.0001$ & $<.0001$ & & & & & & \\
\hline & $\psi$ & -7.96328 & 1.465437 & 0.717 & $2.48 \times 10^{-41}$ & -32.4767 & & & & & \\
\hline \multirow[t]{3}{*}{ 6) } & $\varepsilon$ & 0.4061 & 0.1289 & 0.0430 & $1.22 \times 10^{-37}$ & 1703.3 & & & & & \\
\hline & $\infty$ & $<.0001$ & $<.0001$ & $<.0001$ & 0.9998 & 0.9850 & & & & & \\
\hline & $\Psi$ & -9.45435 & 1.937055 & 0.846819 & 0.000077 & 1.656325 & 1.149874 & & & & \\
\hline \multirow[t]{3}{*}{ 7) } & E & 0.0498 & 0.0136 & 0.0102 & $2.368 \times 10^{-6}$ & 0.0143 & 0.0114 & & & & \\
\hline & $\infty$ & $<.0001$ & $<.0001$ & $<.0001$ & $<.0001$ & $<.0001$ & $<.0001$ & & & & \\
\hline & $\Psi$ & & & & 1.28373 & -1.16511 & 1.125006 & & & & 1.989231 \\
\hline \multirow[t]{3}{*}{ 8) } & $\varepsilon$ & & & & 0.0564 & 0.1041 & 0.081 & & & & 0.00968 \\
\hline & $\infty$ & & & & $<.0001$ & $<.0001$ & $<.0001$ & & & & $<.0001$ \\
\hline & $\Psi$ & -10.0139 & 2.18313 & 0.737407 & & $8.258 \times 10^{-6}$ & $4.1 \times 10^{-5}$ & $3.1 \times 10^{-5}$ & 0.048418 & 0.75214 & \\
\hline \multirow[t]{2}{*}{ 9) } & $\varepsilon$ & 0.0382 & 0.00911 & 0.00601 & & $4.041 \times 10^{-7}$ & $3.164 \times 10^{-7}$ & $4.757 \times 10^{-7}$ & 0.00202 & 0.0118 & \\
\hline & $\infty$ & $<.0001$ & $<.0001$ & $<.0001$ & & $<.0001$ & $<.0001$ & $<.0001$ & $<.0001$ & $<.0001$ & \\
\hline
\end{tabular}

Nota: $\Psi$ : valor de los parámetros $\varepsilon$ : error estándar de los parámetros; $\infty$ : nivel de significancia de los parámetros.

Elaboración propia.

DISCUSIÓN

El ajuste simultaneo de los estimadores de los parámetros de los modelos de volumen y ahusamiento a través de máxima verosimilitud con información completa (FIML) es más eficiente y preciso que la estimación independiente de los parámetros de cada ecuación. Este método minimiza los errores en forma conjunta, maximizando la significancia de los coeficientes de las ecuaciones (Borders, 1989; Fang et al., 2000). En relación con los modelos compatibles de volumen comercial-ahusamiento estudiados, los modelos de Fang et al. (2000) y Cruz-Cobos et al. (2008) fueron los que presentaron el mejor ajuste.
El sistema compatible de ahusamiento y volumen desarrollado por Fang et al. (2000), por su flexibilidad de adaptación a las diversas formas del fuste de los árboles, tiene un ajuste significativo a la base de datos (Diéguez-Aranda, Castedo-Dorado, ÁlvarezGonzález, \& Rojo, 2006).

Sin embargo, el modelo de ahusamiento CruzCobos et al. (2008), aunque simple en su estructura, presenta un buen ajuste. Los resultados de este estudio son congruentes por los reportados para otras especies como Pinus cooperi (Corral-Rivas, DiéguezAranda, Corral-Rivas, \& Castedo-Dorado, 2007; CruzCobos et al., 2008), P. engelmannii (Corral-Rivas et al., 2007; Quiñones-Barraza, De los Santos-Posadas, 
Tabla 7

Datos descriptivos de los modelos compatibles de volumen comercial-ahusamiento para P. engelmannii

\begin{tabular}{|c|c|c|c|c|c|c|c|c|c|c|c|}
\hline $\begin{array}{l}\text { Sistema } \\
\text { de } \\
\text { volumen }\end{array}$ & & $\alpha_{0}$ & $\alpha_{1}$ & $\alpha_{2}$ & $\beta_{0}$ & $\beta_{1}$ & $\beta_{2}$ & $\beta_{3}$ & $\rho_{1}$ & $\rho_{2}$ & $\delta$ \\
\hline & $\psi$ & & & & & -2.02488 & 0.838569 & & & & \\
\hline \multirow[t]{3}{*}{ 1) } & E & & & & & 0.0427 & 0.0330 & & & & \\
\hline & $\infty$ & & & & & $<.0001$ & $<.0001$ & & & & \\
\hline & $\psi$ & -11.0555 & 2.099025 & 1.072114 & 0.00002 & 2.855828 & & & & & \\
\hline \multirow[t]{3}{*}{ 2) } & E & 0.0978 & 0.0305 & 0.0184 & $2.363 \times 10^{-7}$ & 0.00797 & & & & & \\
\hline & $\infty$ & $<.0001$ & $<.0001$ & $<.0001$ & $<.0001$ & $<.0001$ & & & & & \\
\hline & $\psi$ & & & & & 1.160587 & 1.565545 & & & & \\
\hline \multirow[t]{3}{*}{ 3) } & E & & & & & 0.0102 & 0.0260 & & & & \\
\hline & $\infty$ & & & & & $<.0001$ & $<.0001$ & & & & \\
\hline & $\psi$ & -9.94775 & 2.044336 & 0.811806 & 0.331446 & 2.878275 & 2.688267 & & & & \\
\hline \multirow[t]{3}{*}{ 4) } & $\mathrm{E}$ & 0.1387 & 0.0358 & 0.0117 & 0.0493 & 0.00731 & 0.0375 & & & & \\
\hline & $\infty$ & $<.0001$ & $<.0001$ & $<.0001$ & $<.0001$ & $<.0001$ & $<.0001$ & & & & \\
\hline & $\psi$ & -9.85669 & 1.661028 & 1.272564 & 0.000029 & & & & & & \\
\hline \multirow[t]{3}{*}{ 5) } & E & 0.0675 & 0.0172 & 0.0142 & $1.667 \times 10^{-7}$ & & & & & & \\
\hline & $\infty$ & $<.0001$ & $<.0001$ & $<.0001$ & $<.0001$ & & & & & & \\
\hline & $\psi$ & -5.95099 & 1.404914 & 1.204626 & $2.9 \times 10^{-61}$ & -31.2644 & & & & & \\
\hline \multirow[t]{3}{*}{ 6) } & E & 0.5121 & 0.1873 & 0.1828 & $8.56 \times 10^{-57}$ & 6556.7 & & & & & \\
\hline & $\infty$ & $<.0001$ & $<.0001$ & $<.0001$ & 1.000 & 0.9962 & & & & & \\
\hline & $\psi$ & -9.30216 & 1.386614 & 1.420969 & 0.000081 & 1.445024 & 1.333978 & & & & \\
\hline \multirow[t]{3}{*}{ 7) } & E & 0.0795 & 0.0178 & 0.0192 & $5.858 \times 10^{-6}$ & 0.0228 & 0.0249 & & & & \\
\hline & $\infty$ & $<.0001$ & $<.0001$ & $<.0001$ & $<.0001$ & $<.0001$ & $<.0001$ & & & & \\
\hline & $\psi$ & & & & 1.416265 & 0.188865 & 1.464867 & & & & 1.741711 \\
\hline \multirow[t]{3}{*}{ 8) } & E & & & & 0.1039 & 0.2992 & 0.2184 & & & & 0.00914 \\
\hline & $\infty$ & & & & $<.0001$ & 0.2095 & 0.0136 & & & & $<.0001$ \\
\hline & $\psi$ & -9.67148 & 1.819323 & 1.025261 & & $\underset{6}{5.895} \times 10^{-}$ & 0.000033 & 0.018106 & 0.041217 & 0.98885 & \\
\hline \multirow[t]{2}{*}{ 9) } & $\mathrm{E}$ & 0.047 & 0.0123 & 0.0167 & & $2.292 \times 10^{-}$ & $2.314 \times 10$ & 0.0532 & 0.00140 & 0.0 & \\
\hline & $\infty$ & $<.0001$ & $<.0001$ & $<.0001$ & & $<.0001$ & $<.0001$ & $<.0001$ & $<.0001$ & $<.0001$ & \\
\hline
\end{tabular}

Nota: $\Psi$ : valor de los parámetros $\varepsilon$ : error estándar de los parámetros; $\infty$ : nivel de significancia de los parámetros.

Elaboración propia.

Álvarez-González, \& Velázquez-Martínez, 2014), P. patula (Hernández-Pérez, De los Santos-Posadas, Ángeles-Pérez, Valdez-Lazalde, \& Volke- Haller, 2013), P. oocarpa y P. douglasiana (López- Martínez, Cruz-Cobos, Nájera-Luna, \& Hernández, 2015).

\section{CONCLUSIONES}

Los modelos compatibles de volumen comercial ahusamiento se han convertido en una herramienta muy importante para el técnico forestal, ya que con ellos se pueden estimar tanto el volumen total como el volumen comercial; además, en ellos tenemos la 


\section{IIVESTIGAGIÓn Y CUERCIA DE LA UחIVERSIDAD AUTÓNOMA DE AGUASCALIERTES}

integración de ecuaciones de ahusamiento que nos permite estimar diámetros y alturas del fuste.

Aunque el modelo compatible de volumen comercial-ahusamiento de Fang et al. (2000) presentó los mejores resultados para Pinus arizonica, $P$. durangensis y $P$. engelmannii, el sistema de volumen ahusamiento descrito por Cruz-Cobos et al. (2008) es recomendable por su simplicidad.

\section{REFERENCIAS}

- Borders, B. E. (1989). Systems of equations in forest stand modeling. Forest Science, 35(2), 548-556.

- Clutter, J. L. (1980). Notes: Development of taper functions from variable-top merchantable volume equations. Forest Science, 26(1), 117-120. Fortson, J. C., Pienaar, L. V., Brister, G. H., \& Bailey, R. L. (1983). Timber management: A quantitative approach (333 pp.). NY: Wiley \& Sons.

- Corral-Rivas, J. J., Diéguez-Aranda, U., Corral-Rivas, S., \& Castedo-Dorado, F. (2007). A merchantable volume system for major pine species in El Salto, Durango (Mexico). Forest Ecology and Management, 238(1-3), 118-129.

- Cruz-Cobos, F., De los Santos-Posadas, H. M., \& ValdezLazalde, J. R. (2008). Sistema compatible de ahusamientovolumen para Pinus cooperi Blanco en Durango, México. Agrociencia, 42(4), 473-485.

- Demaerschalk, J. P. (1972). Converting volume equations to compatible taper equations. Forest Science, 18(3), 241-245.

- Diéguez-Aranda, U., Castedo-Dorado, F., Álvarez-González J. G., \& Rojo, A. (2006). Compatible taper function for Scots pine plantations in northwestern Spain. Canadian Journal of Forest Research, 36(5), 1190-1205.

- Fang, Z., \& Bailey, R. L. (1999). Compatible volume and taper models with coefficients for tropical species on Hainan Island in southern China. Forest Science, 45(1), 85-100.

- Fang, Z., Borders, B. E., \& Bailey, R. L. (2000). Compatible volume-taper models for loblolly and slash pine based on a system with segmented-stem form factors. Forest Science, $46(1), 1-12$.

- Hernández-Pérez, D., De los Santos-Posadas, H. M., ÁngelesPérez, G., Valdez-Lazalde, J. R., \& Volke-Haller, V. H. (2013). Funciones de ahusamiento y volumen comercial para Pinus patula Schltdl. et Cham. en Zacualtipán, Hidalgo. Revista Mexicana de Ciencias Forestales, 4(16), 35-45.
Kozak, A., Munro, D. D., \& Smith, J. H. G. (1969). Taper functions and their application in forest inventory. The Forestry Chronicle, $45(4), 278-283$

- Lenhart, J. D., \& Clutter, J. L. (1971). Cubic-foot yield tables for old-field loblolly pine plantations in the Georgia Piedmont (Report 22, Georgia Forest Research Council [12 pp.]). Georgia: Georgia Forest Research Council.

- López-Martínez, J. C., Cruz-Cobos, F., Nájera-Luna, J. A., \& Hernández, F. J. (2015). Modelos de ahusamiento y volumen comercial para Pinus oocarpa y Pinus douglasiana en la región de Pueblo Nuevo, Durango. Investigación y Ciencia de la Universidad Autónoma de Aguascalientes, 23(64), 47-53.

- Lynch, T. B., Chang, S. T., \& Chandler, J. P. (1992). Estimation of individual tree volume by importance sampling and antithetic variates from the cylindrical shells integral. Canadian Journal of Forest Research, 22 (3), 326-335.

- Pompa-García, M., Corral-Rivas, J. J., Díaz-Vázquez, M. A., \& Martínez-Salvador, M. (2009). Función de ahusamiento y volumen compatible para Pinus arizonica Engelm. en el suroeste de Chihuahua. Revista Ciencia Forestal en México, 34(105), 119-136.

- Quiñones-Barraza, G., De los Santos-Posadas, H. M., ÁlvarezGonzález, J. G., \& Velázquez-Martínez, A. (2014). Sistema compatible de ahusamiento y volumen comercial para las principales especies de Pinus en Durango, México. Agrociencia, 48(5), 553-567.

- Teshome, T. (2005). A ratio method for predicting stem merchantable volume and associated taper equations for Cupressus Iusitanica, Ethiopia. Forest Ecology and Management, 204(2-3), 171-179.

- Valdez-Lazalde, R., \& Lynch, T. B. (2000). Ecuaciones para estimar volumen comercial y total en rodales aclareados en Pinus patula en Puebla, México. Agrociencia, 34(6), 747-758. 